\title{
Earlier nephrology consultation may not be associated with improved short-term survival of acute kidney injury in very elderly men
}

This article was published in the following Dove Press journal:

Clinical Interventions in Aging

19 December 2016

Number of times this article has been viewed

\author{
Qinglin $\mathrm{Li}^{1}$ \\ Meng Zhao ${ }^{2}$ \\ Jing $\mathrm{Du}{ }^{\prime}$ \\ Xiaodan Wang' \\ 'Department of Geriatric Nephrology, \\ ${ }^{2}$ Department of Clinical Data \\ Repository, Chinese People's \\ Liberation Army General Hospital, \\ Beijing, China
}

Objectives: A delayed nephrology consultation (NC) may be associated with a poor prognosis in acute kidney injury (AKI) patients. The aims of this study were to compare the clinical and laboratory characteristics of elderly AKI patients evaluated and not evaluated by nephrologists and to generate a hypothesis regarding the relationship between the timing of the NC and 90-day outcomes.

Methods: From 2007 to 2015, this study explored associations among the presence and timing of $\mathrm{NC}$ with the non-intensive care unit stay and 90-day mortality in elderly AKI patients at the Geriatric Department of the Chinese People's Liberation Army General Hospital. Early NC and delayed NC were defined as NCs performed before and 2 days after the day of AKI diagnosis, respectively. Multivariable logistic regression was used to adjust for confounding and selection bias.

Results: In total, 623 patients were included for the final analysis, of whom 162 (26\%) were evaluated by nephrologists. The 90 -day mortality rate was $33.2 \%$, and dialysis was required in $1.4 \%$ of patients (9/623). Multivariable analysis showed that a higher prevalence of preexisting chronic obstructive pulmonary disease, AKI diagnosis time, peak serum creatinine level, blood urea nitrogen level, AKI stage, and mortality was associated with the NC. The NC was delayed $(>48 \mathrm{~h})$ in 59 patients $(36.4 \%$ ) (median time to consultation, 4 days). The median AKI diagnosis time, presence of oliguria, uric acid level, and a more severe AKI stage were associated with delayed consultation. Moreover, delayed consultation presented a similar 90-day mortality rate to that of an early NC (50.8\% vs $44.7 \%$, respectively, $P=0.448)$.

Conclusion: In very elderly AKI patients, those evaluated by nephrologists have more severe AKI and a higher mortality rate than those not evaluated by nephrologists. An earlier NC may not be associated with improved 90-day survival.

Keywords: acute kidney injury, very elderly, mortality, nephrology consultation, risk factors, outcomes

\section{Introduction}

Acute kidney injury (AKI), previously termed acute renal failure, is characterized by a sudden decrease in renal function, primarily described in recent years using the widely accepted 2012 Kidney Disease Improving Global Outcomes (KDIGO) classification based on changes in the serum creatinine ( $\mathrm{SCr}$ ) level and/or urine output. ${ }^{1} \mathrm{AKI}$ is more frequent in the elderly, and a relationship between AKI incidence and age is evident. ${ }^{2-6}$ However, few studies have specifically examined AKI in the geriatric population, despite the increasing elderly population and its higher chances of developing AKI.

Despite significant improvements in health care technology in recent years, the mortality of AKI patients remains unacceptably elevated. ${ }^{7-9}$ One reason for this
Correspondence: Xiaodan Wang Department of Geriatric Nephrology, Chinese People's Liberation Army General Hospital, 28 Fuxing Road, 100853, Beijing, China

Email xdwang I I @@hotmail.com 
phenomenon is that the SCr level alone is a relatively late and imprecise biomarker of kidney dysfunction, which may also lead to a delayed diagnosis, especially in the elderly population. ${ }^{10,11}$

Clinical studies have shown that a timely nephrology consultation (NC) could potentially have many benefits such as allowing earlier identification and modification of AKI patients' outcomes. For example, Mehta et al concluded that a delayed NC was associated with increased mortality among dialyzed and nondialyzed patients in the intensive care unit (ICU). ${ }^{12}$ Recent studies have also indicated that an early NC may improve hospital-acquired AKI prognosis, ${ }^{12-16}$ although few studies have examined the effect of an early NC on outcomes in very elderly patients ( $\geq 75$ years) or diagnosing AKI using KDIGO criteria.

The aims of this study were to compare the clinical and laboratory characteristics of elderly AKI patients evaluated and not evaluated by nephrologists and to generate a hypothesis of the relationship between the timing of the $\mathrm{NC}$ and 90-day outcomes.

\section{Study population and methods}

This was a retrospective cohort study. All elderly patients ( $\geq 75$ years) who were admitted to the Geriatric Department of the Chinese People's Liberation Army (PLA) General Hospital between January 2007 and December 2015 were evaluated for AKI during their hospital stay. The study design was approved by the Clinical Ethics Committee of the Chinese PLA General Hospital, and each participant provided written informed consent. All AKI patients were followed up for 90 days after AKI diagnosis or until death.

Clinical data were noted, including the demographic profile (age, gender, and body mass index) and comorbidities (history of hypertension, coronary disease, chronic obstructive pulmonary disease [COPD], and diabetes mellitus), time of AKI diagnosis, etiology of AKI (induced by infection, hypovolemia, cardiovascular events, nephrotoxic drugs, surgery, or uncertain causes), need for dialysis, need for mechanical ventilation (MV), urine output, and mean aortic pressure. Other laboratory data evaluated included baseline levels of $\mathrm{SCr}, \mathrm{SCr}$ at AKI diagnosis, peak SCr, blood urea nitrogen (BUN), uric acid, serum prealbumin, albumin, kalemia, serum calcium, serum magnesium, serum phosphate, and hemoglobin.

AKI was diagnosed exclusively based on SCr levels, that is, an $\mathrm{SCr}$ increase $\geq 0.3 \mathrm{mg} / \mathrm{dL}(\geq 26.5 \mu \mathrm{mol} / \mathrm{L})$ within $48 \mathrm{~h}$, or an increase to $\geq 1.5$-fold above the baseline value within the prior 7 days. ${ }^{1}$ The KDIGO criteria were used. AKI severity was scored according to the difference between the $\mathrm{SCr}$ baseline and peak values, using the KDIGO staging criteria: stage 1 , an increase $\geq 26.5 \mu \mathrm{mol} / \mathrm{L}$, or an increase $\geq 1.5$ - to 1.9 -fold above the baseline value; stage 2 , an increase to $\geq 2$ - to 2 .9-fold above the baseline value; and stage 3 , an increase to $\geq 3$-fold above the baseline value, an increase to $\geq 353.6 \mu \mathrm{mol} / \mathrm{L}$, or initiation of renal replacement therapy. Urine output was not available in the cohort, and the KDIGO urine output criteria could not be applied.

The baseline SCr level was the most recent stable measure obtained 3 months prior to hospital admission for AKI. ${ }^{5}$ The peak SCr was the highest SCr level reached in the episode. Estimated glomerular filtration rates (eGFRs) were calculated by the Chronic Kidney Disease Epidemiology Collaboration (CKD-EPI) method. ${ }^{17}$ Oliguria was defined as urinary output $<400 \mathrm{~mL} / 24 \mathrm{~h}$.

The exclusion criteria were age $<75$ years, patients with a history of CKD of any stage, ${ }^{18}$ hospital stay of $<48 \mathrm{~h}$, those who had no SCr or only one SCr examination, those with missing or incomplete medical history, and those with early death in $<48 \mathrm{~h}$ after admission. Females were also excluded, because fewer females were treated than males during the study period.

All elderly AKI patients were divided into two groups: those evaluated and not evaluated by nephrologists. Thereafter, AKI patients evaluated by nephrologists were further divided into two subgroups (early and delayed consultation) based on the time to consultation from the day of laboratory diagnosis of AKI. An early NC was defined as $\leq 48 \mathrm{~h}$ and a delayed NC was defined as $>48 \mathrm{~h}$.

The primary outcome was 90-day mortality. Clinical and laboratory characteristics were also recorded.

\section{Statistical analysis}

Continuous variables are presented as the mean \pm standard deviations, or medians ( $25 \%-75 \%$ interquartile range), depending on the variable distribution. Discrete variables are presented as counts or percentages. Between-group comparisons were performed using Student's $t$-test or the Mann-Whitney $U$-test. Correlations between potential factors were assessed using Pearson's chi-squared or Fisher's exact test. Multivariable logistic regression models were constructed with forward variable selection, with the exit criterion set at $P<0.25$. A $P$-value $<0.05$ was considered to reflect statistical significance. Statistical analyses were performed using Statistical Package for the Social Sciences (SPSS) Version 17.0 for Windows (SPSS Inc., Chicago, IL, USA). 


\section{Results}

Among 1,711 study patients, 668 (39\%) developed AKI. Only 45 patients were excluded, resulting in 623 AKI patients suitable for analysis. The study flowchart is presented in Figure 1. The median age of the cohort was 87 years. The median baseline $\mathrm{SCr}$ level was $74 \mu \mathrm{mol} / \mathrm{L}$, and baseline eGFR was $78.4 \mathrm{~mL} / \mathrm{min} / 1.73 \mathrm{~m}^{2}$. Using the KDIGO criteria, 294 patients (47.2\%) had stage 1 AKI, $157(25.2 \%)$ had stage 2 AKI, and 172 (27.6\%) had stage 3 AKI. Of the 623 patients, $78.2 \%$ had coronary disease, $73.8 \%$ had hypertension, $70.3 \%$ had COPD, and 35.8\% had diabetes mellitus. An NC was performed in 26\% (162/623) of AKI patients and occurred 2 (1-4) days after the day of AKI diagnosis. Most patients referred to nephrologists were subjected to an early NC (63.6\%). The overall 90-day mortality was 33.2\% (207/623), and dialysis was required in only $1.4 \%(9 / 623)$ of patients.

\section{NC}

As shown in Table 1, NC patients were older (median age: 88 vs 87 years, $P=0.115$ ), and the majority of these patients had COPD (79\% vs $67.2 \%, P=0.005)$. Of the $162(26 \%)$ patients referred to nephrologists, AKI diagnosis occurred after 2 days ( $1-5$ days), compared to 4 days (2-7 days) for patients not evaluated by nephrologists $(P=0.001)$. The median SCr level (70 vs $75 \mu \mathrm{mol} / \mathrm{L}, P=0.010)$ and eGFR ( $80.1 \mathrm{vs} 78 \mathrm{~mL} / \mathrm{min} / 1.73 \mathrm{~m}^{2}, P=0.032$ ) at baseline were significantly different between the two groups.

As shown in Table 1, patients evaluated by nephrologists presented higher mortality rate $(46.9 \%$ vs $28.4 \%, P<0.001)$, with more patients in KDIGO stage 3, and fewer patients in stage 1 (51.9\% vs $19.1 \%, 22.8 \%$ vs $55.7 \%$; $P<0.001)$. Oliguria (9.9\% vs $4.1 \%, P=0.006)$, dialysis $(5.6 \%$ vs $0 \%$, $P<0.001)$, requiring $\mathrm{MV}(51.9 \%$ vs $31.9 \%, P<0.001)$, anemia (109 \pm 23 vs $113 \pm 22 \mathrm{~g} / \mathrm{L}, P=0.013)$, a lower prealbumin level (168 vs $186 \mathrm{~g} / \mathrm{L}, P=0.011$ ), and hypoalbuminemia $(33 \pm 5.2$ vs $34.8 \pm 5.5 \mathrm{~g} / \mathrm{L}, P=0.001)$ were more frequent among patients referred to nephrologists. NC patients had significantly higher uric acid (399.2 vs $360 \mu \mathrm{mol} / \mathrm{L}, P=0.005)$, kalemia (4.3 vs $4.1 \mathrm{mmol} / \mathrm{L}, P=0.035$ ), and phosphate levels ( $1.3 \mathrm{vs} 1.2 \mathrm{mmol} / \mathrm{L}, P=0.031$ ). As expected, patients evaluated by nephrologists had higher $\mathrm{SCr}, \mathrm{BUN}$, and peak $\mathrm{SCr}$ levels at the time of AKI diagnosis (all $P<0.001$ ), and the presence or absence of an NC was associated with 90-day mortality (Figure 2).

Multivariate logistic regression analysis revealed that an $\mathrm{NC}$ was significantly associated with a higher prevalence of

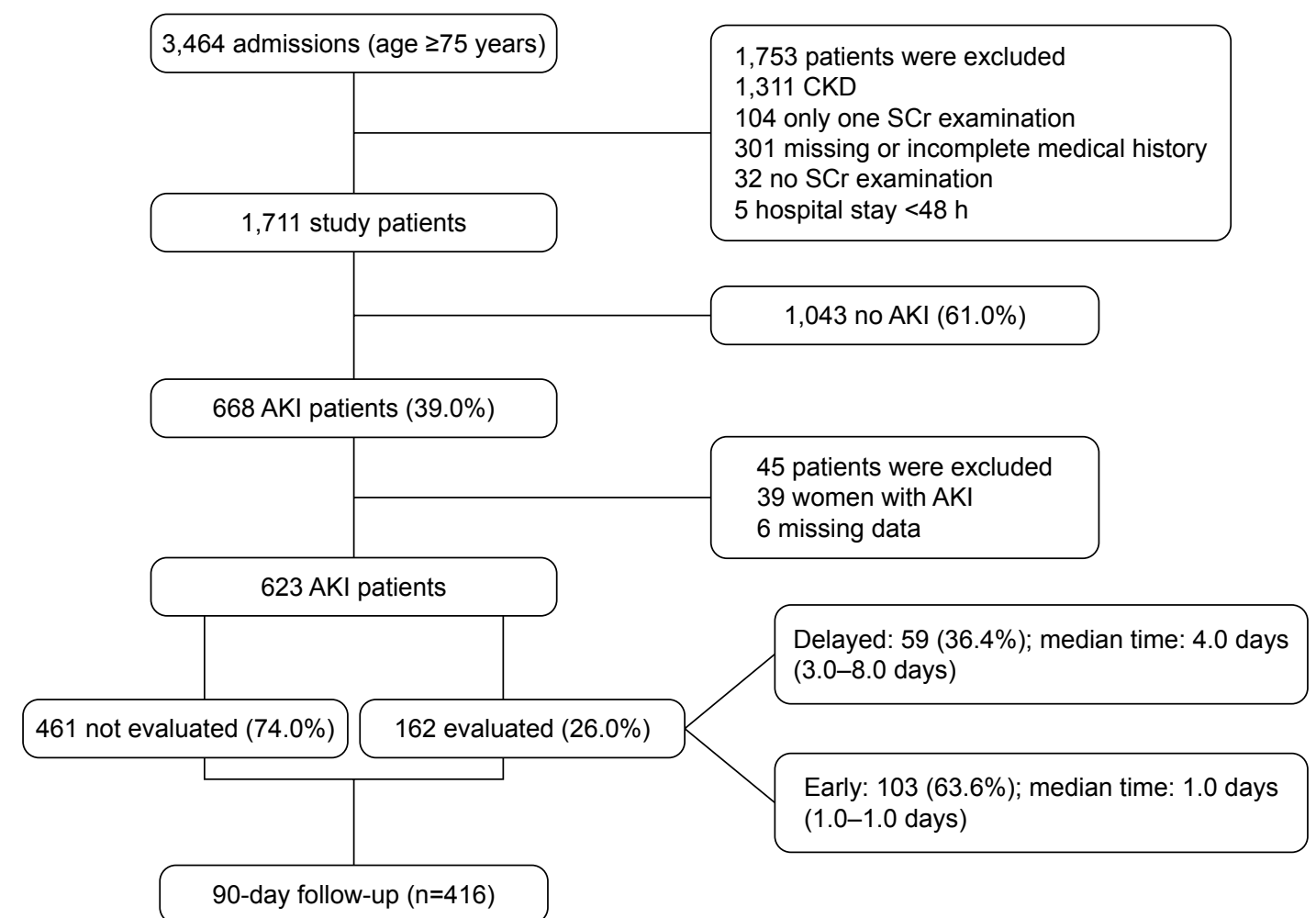

Figure I Flowchart of patients included and excluded in the study.

Abbreviations: $\mathrm{AKI}$, acute kidney injury; $\mathrm{CKD}$, chronic kidney disease; $\mathrm{SCr}$, serum creatinine. 
Table I Comparisons of the clinical characteristics between elderly AKI patients with and without an NC

\begin{tabular}{|c|c|c|c|c|}
\hline Characteristics & $\begin{array}{l}\text { AKI patients } \\
(n=623)\end{array}$ & $\begin{array}{l}\text { NC } \\
(n=162)\end{array}$ & $\begin{array}{l}\text { No NC } \\
(n=46 I)\end{array}$ & $P$-value \\
\hline Age (years) & 87 (84-91) & $88(84-91)$ & $87(84-91)$ & 0.115 \\
\hline BMI $\left(\mathrm{kg} / \mathrm{m}^{2}\right)$ & $23.0 \pm 3.1$ & $23.0 \pm 2.9$ & $23.0 \pm 3.2$ & 0.658 \\
\hline \multicolumn{5}{|l|}{ Comorbidity } \\
\hline Coronary disease & $487(78.2)$ & $129(79.6)$ & $358(77.7)$ & 0.601 \\
\hline Hypertension & $460(73.8)$ & |2| (74.7) & $339(73.5)$ & 0.773 \\
\hline COPD & $438(70.3)$ & $128(79)$ & $310(67.2)$ & 0.005 \\
\hline Diabetes & $223(35.8)$ & $57(35.2)$ & $166(36.0)$ & 0.851 \\
\hline Baseline SCr $(\mu \mathrm{mol} / \mathrm{L})$ & $74.0(62.0-84.0)$ & $70.0(60.0-81.3)$ & $75.0(63.0-84.5)$ & 0.010 \\
\hline Baseline eGFR $\left(\mathrm{mL} / \mathrm{min} / \mathrm{I} .73 \mathrm{~m}^{2}\right)$ & $78.4(71.3-84.9)$ & $80.1(72.7-85.9)$ & $78.0(70.6-84.7)$ & 0.032 \\
\hline \multicolumn{5}{|l|}{ Etiology of AKI } \\
\hline Infections & $247(39.6)$ & $67(41.4)$ & $180(39.0)$ & 0.605 \\
\hline Hypovolemia & I $48(23.8)$ & $32(19.8)$ & $116(25.2)$ & 0.164 \\
\hline Cardiovascular events & $99(15.9)$ & $29(17.9)$ & $70(15.2)$ & 0.416 \\
\hline Nephrotoxicity & $75(12)$ & $19(11.7)$ & $56(12.1)$ & 0.888 \\
\hline Surgery & $44(7.1)$ & II (6.8) & $33(7.2)$ & 0.875 \\
\hline Others & $10(1.6)$ & $4(2.5)$ & $6(1.3)$ & 0.513 \\
\hline AKI diagnosis time (days) & $2.0(2.0-7.0)$ & $2.0(1.0-5.0)$ & $4.0(2.0-7.0)$ & 0.001 \\
\hline \multicolumn{5}{|c|}{ Parameter at the time of AKI diagnosis } \\
\hline MAP $(\mathrm{mmHg})$ & $78 \pm 14$ & $79 \pm 13$ & $78 \pm 15$ & 0.977 \\
\hline Oliguria & $35(5.6)$ & $16(9.9)$ & $19(4.1)$ & 0.006 \\
\hline Dialysis & $9(1.4)$ & $9(5.6)$ & 0 & $<0.001$ \\
\hline MV & $231(37.1)$ & $84(51.9)$ & $147(31.9)$ & $<0.00 \mathrm{I}$ \\
\hline \multicolumn{5}{|c|}{ Laboratory results at the time of $\mathrm{AKI}$ diagnosis } \\
\hline $\mathrm{SCr}(\mu \mathrm{mol} / \mathrm{L})$ & $132.0(|| 8.2-147.0)$ & $138.0(\mid 27.2-160.5)$ & I28.| (| |6.2-143.6) & $<0.00 \mathrm{I}$ \\
\hline Peak SCr $(\mu \mathrm{mol} / \mathrm{L})$ & I 44.0 (I24.8-204.0) & $206.5(143.3-353.7)$ & $137.8(|2| .2-173.3)$ & $<0.00$ I \\
\hline BUN (mmol/L) & $12.8(8.9-21.3)$ & $16.7(10.7-26.8)$ & II.8 (8.4-20.1) & $<0.00 \mathrm{I}$ \\
\hline Uric acid $(\mu \mathrm{mol} / \mathrm{L})$ & $367.0(293.7-471.0)$ & $399.2(312.9-506.7)$ & $360.0(285.0-457.5)$ & 0.005 \\
\hline Prealbumin $(g / L)$ & 181 (139-234) & $168(125-227)$ & $186(143-239)$ & 0.011 \\
\hline Albumin $(g / L)$ & $34.3 \pm 5.5$ & $33.0 \pm 5.2$ & $34.8 \pm 5.5$ & 0.001 \\
\hline Kalemia (mmol/L) & $4.1(3.8-4.7)$ & $4.3(3.8-4.9)$ & $4.1(3.8-4.6)$ & 0.035 \\
\hline Magnesium (mmol/L) & $0.9(0.8-1.0)$ & $0.9(0.8-1.0)$ & $0.9(0.8-1.0)$ & 0.972 \\
\hline Calcium (mmol/L) & $2.2(2.1-2.3)$ & $2.2(2.0-2.3)$ & $2.2(2.1-2.4)$ & 0.428 \\
\hline Phosphate (mmol/L) & $1.2(1.0-1.4)$ & $1.3(1.0-1.6)$ & $1.2(0.9-1.4)$ & 0.031 \\
\hline Hemoglobin $(g / L)$ & $112 \pm 23$ & $109 \pm 23$ & $113 \pm 22$ & 0.013 \\
\hline AKI stage & & & & $<0.001$ \\
\hline I & $294(47.2)$ & $37(22.8)$ & $257(55.7)$ & \\
\hline 2 & $157(25.2)$ & $41(25.3)$ & $116(25.2)$ & \\
\hline 3 & $172(27.6)$ & $84(51.9)$ & $88(19.1)$ & \\
\hline Mortality rate & $207(33.2)$ & $76(46.9)$ & |3| (28.4) & $<0.00 \mathrm{I}$ \\
\hline
\end{tabular}

Notes: Values are $\mathrm{n}(\%)$, mean $\pm \mathrm{SD}$ or median (interquartile range). $1 \mathrm{mmHg}=0.133 \mathrm{kPa}$.

Abbreviations: AKI, acute kidney injury; NC, nephrology consultation; BMI, body mass index; COPD, chronic obstructive pulmonary disease; SCr, serum creatinine; eGFR, estimated glomerular filtration rate; $\mathrm{MAP}$, mean aortic pressure $(\mathrm{I} \mathrm{mmHg}=0.133 \mathrm{kPa})$; $\mathrm{MV}$, mechanical ventilation; $\mathrm{BUN}$, blood urea nitrogen; SD, standard deviation.

preexisting COPD (odds ratio [OR] $=1.794 ; 95 \%$ confidence interval $[\mathrm{CI}]: 1.118-2.879 ; P=0.015)$, AKI diagnosis time (OR $=0.888 ; 95 \%$ CI: 0.812-0.972; $P=0.010)$, peak $\mathrm{SCr}$ level (OR $=1.006 ; 95 \%$ CI: 1.003-1.008; $P<0.001)$, BUN level $(\mathrm{OR}=1.022 ; 95 \% \mathrm{CI}: 1.002-1.043 ; P=0.033)$, AKI stage $(\mathrm{OR}=1.472 ; 95 \% \mathrm{CI}: 1.021-2.122 ; P=0.038)$, and mortality $(\mathrm{OR}=0.531 ; 95 \%$ CI: 0.303-0.931; $P=0.027)$ (Table 2$)$.

\section{NC timing - factors associated with a delayed $\mathrm{NC}$}

Several baseline differences were noted between the early and delayed consultation groups (Table 3 ). Of the 59 (35.6\%) patients with delayed consultation, AKI diagnosis occurred after 2 days (2-7 days), while the median time to consultation was 4 days (3-8 days). Delayed NC patients were more likely to be older (median age: 89 vs 88 years, $P=0.207$ ) and to have a lower median eGFR (78.6 vs $81.4 \mathrm{~mL} / \mathrm{min} / 1.73 \mathrm{~m}^{2}$, $P=0.233$ ) than the early NC group. They had higher peak $\mathrm{SCr}$ (227 vs $183 \mu \mathrm{mol} / \mathrm{L}, P=0.180$ ), lower uric acid (361.7 vs $419 \mu \mathrm{mol} / \mathrm{L}, P=0.004)$, lower kalemia (4.1 vs $4.4 \mathrm{mmol} / \mathrm{L}$, $P=0.140$ ), and lower phosphate ( $1.2 \mathrm{vs} 1.3 \mathrm{mmol} / \mathrm{L}, P=0.031$ ) levels at the time of AKI diagnosis. Accordingly, the prevalence of oliguria was significantly lower in the delayed $\mathrm{NC}$ group ( $1.7 \%$ vs $14.6 \%, P=0.008)$. 


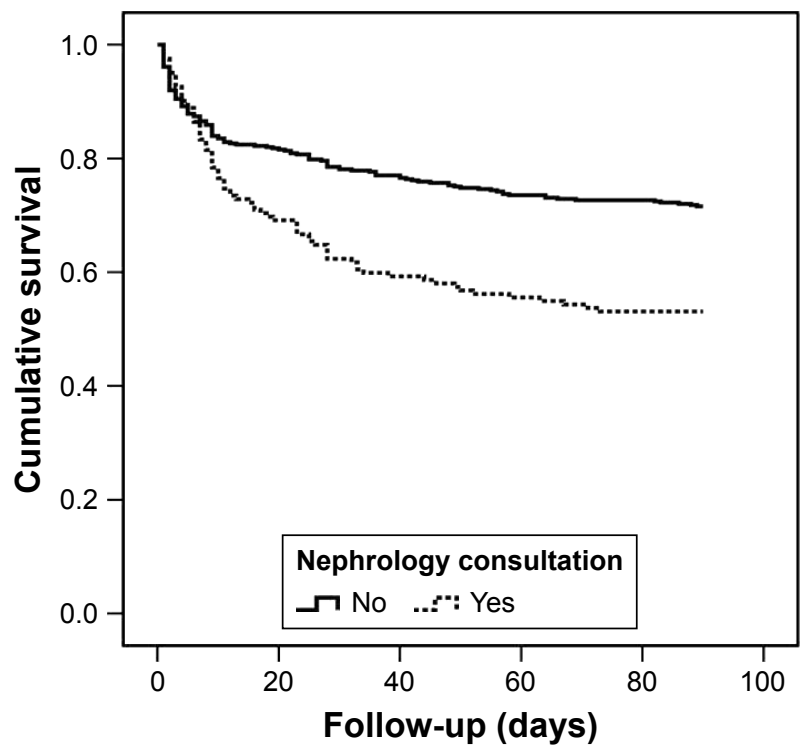

Figure 2 Kaplan-Meier survival curves for 90 -day mortality of $\mathrm{AKI}$ in patients with and without a nephrology consultation (log-rank test: $P<0.001$ ).

Abbreviation: AKI, acute kidney injury.

Multivariate logistic regression analysis revealed that a delayed $\mathrm{NC}$ was significantly associated with AKI diagnosis time $(\mathrm{OR}=1.269 ; 95 \% \mathrm{CI}: 1.078-1.495 ; P=0.004)$, oliguria (OR $=0.116 ; 95 \%$ CI: $0.014-0.952 ; P=0.045)$, uric acid level (OR $=0.996 ; 95 \%$ CI: 0.994-0.999; $P=0.009$ ), and a more severe AKI stage (OR $=3.365$; $95 \%$ CI: $1.297-8.733$; $P=0.013$ ) (Table 4).

\section{Influence of a delayed NC on patient outcomes}

As shown in Table 3, more patients were classified as KDIGO stage 3 and fewer patients were classified as stage 1 and stage $2(57.6 \%$ vs $48.5 \%, 18.6 \%$ vs $25.2 \%, 23.7 \%$ vs $26.2 \% ; P=0.238)$. Dialysis was necessary in $5.1 \%$ of the delayed group, compared to $5.8 \%$ of the early group $(P=1.000)$. Surprisingly, delayed consultation was not associated with increased mortality ( $50.8 \%$ vs $44.7 \%, P=0.448)$ (Figure 3).

Table 2 Associations (with $P<0.25$ ) between AKI patients characteristics and NC

\begin{tabular}{llll}
\hline Risk factors & OR & $\mathbf{9 5 \% ~ C l}$ & $P$-value \\
\hline Presence of COPD & 1.794 & $1.118-2.879$ & 0.015 \\
AKI diagnosis time & 0.888 & $0.812-0.972$ & 0.010 \\
Peak SCr level & 1.006 & $1.003-1.008$ & $<0.001$ \\
BUN level & 1.022 & $1.002-1.043$ & 0.033 \\
AKI stage & 1.472 & $1.021-2.122$ & 0.038 \\
Mortality & 0.531 & $0.303-0.931$ & 0.027 \\
\hline
\end{tabular}

Abbreviations: $\mathrm{AKI}$, acute kidney injury; $\mathrm{NC}$, nephrology consultation; $\mathrm{SCr}$, serum creatinine; $\mathrm{BUN}$, blood urea nitrogen; $\mathrm{Cl}$, confidence interval; OR, odds ratio.

\section{Discussion}

It is widely recognized that the timing of an $\mathrm{NC}$ is associated with AKI patient outcomes. However, few studies have specifically examined this important topic with regard to the very elderly population. This analysis was restricted to patients who developed AKI, compared to the clinical and laboratory characteristics of patients evaluated and not evaluated by nephrologists, and generated a hypothesis regarding the relationship between the timing of the NC and short-term outcomes. In this observational study, 39\% of patients developed AKI, and fewer patients (26\%) were evaluated by nephrologists. These patients presented higher mortality than those not evaluated, most likely due to their more severe illness characteristics (more patients with oliguria, greater need for dialysis, higher SCr and BUN levels, and more stage 3 patients).

In the present study, 59 of the NCs $(36.4 \%)$ were delayed $(>48 \mathrm{~h}$ ), and a delayed consultation was not associated with increased mortality. If this association is valid, it can be inferred that consultation timing itself is not responsible for the decreased mortality risk in elderly AKI patients.

Two recently published studies have evaluated the association between the timing of the $\mathrm{NC}$ and patient outcomes. ${ }^{13,19}$ The retrospective observational study by Ponce et al enrolled 148 AKI patients (Acute Kidney Injury Network criteria) treated in the ICU, ${ }^{13}$ of whom only 77 were evaluated by nephrologists. Patients in the early NC group had a mean SCr level of $6.6 \pm 1.8 \mathrm{mg} / \mathrm{dL}$, compared to a mean SCr level of $4.2 \pm 2.4 \mathrm{mg} / \mathrm{dL}$ in the delayed group. A delayed NC, using a $48 \mathrm{~h}$ interval, was associated with increased mortality after adjustment for multivariable analysis. In a study by Costa e Silva et al, ${ }^{19}$ which was a prospective observational study including 366 critically ill AKI patients (RIFLE [Risk, Injury, Failure, Loss and End-stage Kidney Disease] criteria), 31.4\% (155/366) of patients underwent dialysis, and hospital mortality occurred in $67.8 \%$ (248/366). The authors also reported that delayed $\mathrm{NC}$ was associated with higher mortality and increased dialysis dependence rates at hospital discharge.

There are several potential reasons why only $26 \%$ of the AKI patients were evaluated by nephrologists. One explanation may be the definition of AKI, which is neither uniformly known nor accepted in the non-nephrologic community. ${ }^{15}$ Although the KDIGO criteria provide a unique basis for epidemiologic and interventional outcome studies and suggest increasing $\mathrm{SCr}$ and urine volume monitoring in patients with a high risk for AKI, these criteria are not commonly used in the clinical setting. Moreover, the SCr level has a nonlinear relationship with the eGFR and requires time to 
Table 3 AKI patients characteristics according to early and delayed NC

\begin{tabular}{|c|c|c|c|c|}
\hline Characteristics & $\begin{array}{l}\text { NC patients } \\
(n=162)\end{array}$ & $\begin{array}{l}\text { Delayed NC } \\
(n=59)\end{array}$ & $\begin{array}{l}\text { Early NC } \\
(n=103)\end{array}$ & $P$-value \\
\hline Age (years) & $88.0(84.0-91.0)$ & $89.0(85.0-91.0)$ & $88.0(84.0-91.0)$ & 0.207 \\
\hline BMI $\left(\mathrm{kg} / \mathrm{m}^{2}\right)$ & $23.0 \pm 2.9$ & $22.8 \pm 3.0$ & $23.0 \pm 2.9$ & 0.474 \\
\hline \multicolumn{5}{|l|}{ Comorbidity } \\
\hline Coronary disease & $129(79.6)$ & $47(79.7)$ & $82(79.6)$ & 0.994 \\
\hline Hypertension & I2I (74.7) & $45(76.3)$ & $76(73.8)$ & 0.726 \\
\hline COPD & $128(79.0)$ & $46(78.0)$ & $82(79.6)$ & 0.805 \\
\hline Diabetes & $57(35.2)$ & 20 (33.9) & 37 (35.9) & 0.795 \\
\hline Baseline SCr $(\mu \mathrm{mol} / \mathrm{L})$ & $70.0(60.0-81.3)$ & $70.0(63.0-82.0)$ & $70.0(58.0-80.0)$ & 0.327 \\
\hline Baseline eGFR $\left(\mathrm{mL} / \mathrm{min} / \mathrm{l} .73 \mathrm{~m}^{2}\right)$ & $80.1(72.7-85.9)$ & $78.6(71.3-85.9)$ & $81.4(74.1-85.9)$ & 0.233 \\
\hline \multicolumn{5}{|l|}{ Etiology of AKI } \\
\hline Sepsis & $67(4 I .4)$ & $25(42.4)$ & $42(40.8)$ & 0.843 \\
\hline Ischemia & $32(19.8)$ & II (I8.6) & $21(20.4)$ & 0.788 \\
\hline Cardiovascular event & $29(17.9)$ & $12(20.3)$ & $17(16.5)$ & 0.540 \\
\hline Nephrotoxicity & $19(11.7)$ & $8(13.6)$ & II (I0.7) & 0.584 \\
\hline Surgery & II (6.8) & $2(3.4)$ & $9(8.7)$ & 0.328 \\
\hline Others & $4(2.5)$ & $\mathrm{I}(\mathrm{I} .7)$ & $3(2.9)$ & 1.000 \\
\hline AKI diagnosis time (days) & $2.0(1.0-5.0)$ & $2.0(2.0-7.0)$ & $2.0(1.0-4.0)$ & 0.004 \\
\hline \multicolumn{5}{|c|}{ Parameter at the time of $\mathrm{AKI}$ diagnosis } \\
\hline MAP $(\mathrm{mmHg})$ & $79 \pm 13$ & $78 \pm 12$ & $79 \pm 14$ & 0.969 \\
\hline Oliguria & $16(9.9)$ & $\mathrm{I}(\mathrm{I} .7)$ & $15(14.6)$ & 0.008 \\
\hline Dialysis & $9(5.6)$ & $3(5.1)$ & $6(5.8)$ & 1.000 \\
\hline MV & $84(51.9)$ & $28(47.5)$ & $56(54.4)$ & 0.397 \\
\hline \multicolumn{5}{|c|}{ Laboratory results at the time of $\mathrm{AKI}$ diagnosis } \\
\hline $\mathrm{SCr}(\mu \mathrm{mol} / \mathrm{L})$ & $138.0(\mid 27.2-160.5)$ & $138.0(125.2-155.0)$ & | $38.0(\mid 28.0-163.0)$ & 0.292 \\
\hline Peak SCr $(\mu \mathrm{mol} / \mathrm{L})$ & $206.5(143.3-353.7)$ & $227.0(150.1-399.3)$ & $183.0(|39.2-34| .3)$ & 0.180 \\
\hline $\mathrm{BUN}(\mathrm{mmol} / \mathrm{L})$ & $16.7(10.7-26.8)$ & I7.| (I0.8-23.4) & I6.I (I0.1-27.6) & 0.746 \\
\hline Uric acid $(\mu \mathrm{mol} / \mathrm{L})$ & $399.2(312.9-506.7)$ & $361.7(279.2-459.0)$ & $419.0(335.0-525.2)$ & 0.004 \\
\hline Prealbumin (g/L) & $168.0(\mid 25.0-227.0)$ & $163.0(120.0-209.0)$ & $169.0(\mid 25.0-230.0)$ & 0.614 \\
\hline Albumin $(g / L)$ & $33.0 \pm 5.2$ & $33.0 \pm 5.0$ & $33.1 \pm 5.4$ & 0.826 \\
\hline Kalemia (mmol/L) & $4.3(3.8-4.9)$ & 4.I (3.8-4.7) & $4.4(3.9-4.9)$ & 0.140 \\
\hline Magnesium (mmol/L) & $0.9(0.8-1)$ & $0.9(0.8-1.0)$ & $0.9(0.8-1.1)$ & 0.943 \\
\hline Calcium (mmol/L) & $2.2(2-2.3)$ & $2.2(2.1-2.4)$ & $2.2(2.0-2.3)$ & 0.261 \\
\hline Phosphate (mmol/L) & $1.3(1-1.6)$ & $1.2(0.9-1.5)$ & $1.3(1.1-1.6)$ & 0.031 \\
\hline Hemoglobin (g/L) & $109 \pm 23$ & $106 \pm 23$ & $1 \mathrm{II} \pm 24$ & 0.382 \\
\hline AKI stage & & & & 0.238 \\
\hline 1 & $37(22.8)$ & II (I8.6) & $26(25.2)$ & \\
\hline 2 & $4 \mid(25.3)$ & $14(23.7)$ & $27(26.2)$ & \\
\hline 3 & $84(51.9)$ & $34(57.6)$ & $50(48.5)$ & \\
\hline Mortality rate & $76(46.9)$ & $30(50.8)$ & $46(44.7)$ & 0.448 \\
\hline
\end{tabular}

Notes: Values are $\mathrm{n}(\%)$, mean $\pm \mathrm{SD}$ or median (interquartile range). I $\mathrm{mmHg}=0.133 \mathrm{kPa}$.

Abbreviations: AKI, acute kidney injury; NC, nephrology consultation; BMI, body mass index; COPD, chronic obstructive pulmonary disease; MAP, mean aortic pressure ( I mmHg =0.133 kPa); MV, mechanical ventilation; SCr, serum creatinine; eGFR, estimated glomerular filtration rate; BUN, blood urea nitrogen; SD, standard deviation.

accumulate, thus contributing to delays in the detection of important changes in kidney function. According to the KDIGO criteria, the $48 \mathrm{~h}$ window for diagnosis does enhance the sensitivity for earlier diagnosis relative to the 7-day window because if the SCr level significantly increases within $48 \mathrm{~h}$, it is more likely to attract the attention of clinicians. In contrast, if the patient's SCr level increases to 1.5 times the baseline value in 7 days, clinicians may not be aware of AKI occurrence due to the "normal SCr" level, especially among elder people with lower baseline $\mathrm{SCr}$ levels. The results also confirmed that the AKI diagnosis time was 2 days in the NC group, compared to 4 days in the group without consultation. Finally, no universal definition for NC timing exists for AKI patients. Elderly patients often had many comorbidities, thus increasing the complexity of medical care and possibly increasing the time required to make treatment decisions.

Several other reasons may explain why early consultation did not improve patient outcomes. First, the study excluded patients with CKD. As CKD is a potent risk factor 
Table 4 Associations (with $P<0.25$ ) between patients characteristics and delayed NC

\begin{tabular}{llll}
\hline Risk factors & OR & $\mathbf{9 5 \%} \mathbf{C l}$ & P-value \\
\hline AKI diagnosis time & 1.269 & $1.078-1.495$ & 0.004 \\
Oliguria & 0.116 & $0.014-0.952$ & 0.045 \\
Uric acid level & 0.996 & $0.994-0.999$ & 0.009 \\
AKI stage 3 & 3.365 & $1.297-8.733$ & 0.013 \\
\hline
\end{tabular}

Abbreviations: $\mathrm{NC}$, nephrology consultation; $\mathrm{AKI}$, acute kidney injury; $\mathrm{Cl}$, confidence interval; OR, odds ratio.

for mortality, excluding patients with CKD in this analysis may have potentially underestimated the impact of an early $\mathrm{NC}$ on survival. Second, although these elderly patients were treated in non-ICU wards, they had close monitoring and timely treatment by practitioners and had reduced the short-term mortality to a certain extent. In addition, of the 163 stage 3 AKI patients, only nine patients required dialysis during follow-up, and nine were evaluated by nephrologists. Decisions about dialysis initiation in very elderly AKI patients are influenced by several factors: AKI severity, blood vessel volume, electrolyte and acid-base status, urine output, hemodynamics, nutritional status, and attending physicians' preferences. An important issue in the present study is that dialysis cannot be initiated without an $\mathrm{NC}$ because in the hospital, the nephrologist is the only professional who initiates dialysis; so if the patient is not evaluated by a nephrologist, they are not treated with dialysis.

\section{Limitations}

The present study had some potential weaknesses. First, it is a one-center retrospective study, and some potentially

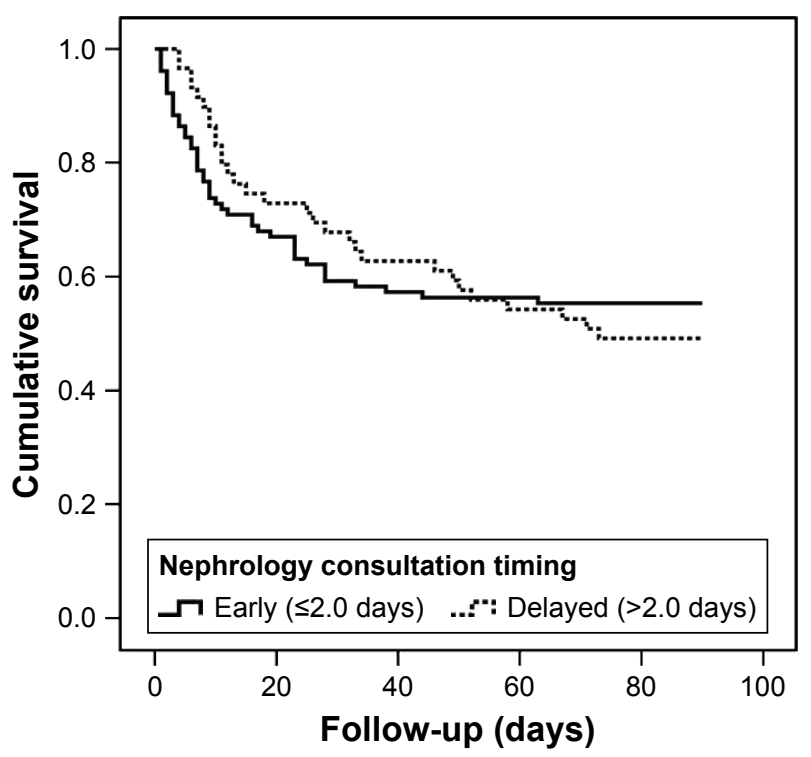

Figure 3 Kaplan-Meier survival curves for 90-day mortality in patients with early and delayed nephrology consultations (log-rank test: $P=0.876$ ). important variables were not included, such as the Acute Physiologic and Chronic Health Evaluation II score and the sequential organ failure assessment score, which can also predict AKI patient outcomes. Second, data from a hospital catering to the elderly were analyzed, and because most patients were male and fewer females were treated in the hospital, females were excluded from the study. Thus, biased results are unavoidable. Third, the definition of AKI in this study was based on $\mathrm{SCr}$ levels. Urine output criteria were not used because these data were incomplete. Fourth, the follow-up duration was short. The study also did not explore the effects of an $\mathrm{NC}$ on kidney outcome.

\section{Conclusion}

In summary, among very elderly patients with AKI, an NC was associated with higher severity of disease (ie, higher peak SCr levels, BUN levels, and AKI stage). As expected, mortality significantly differed between patients who underwent a consultation and those who did not receive a consultation. Fewer AKI patients had a delayed NC (median of 4 days); however, a delayed $\mathrm{NC}$ may not be associated with increased short-term mortality.

\section{Acknowledgments}

This manuscript was edited for English language by American Journal Experts. This study was funded by grants from the National Natural Science Foundation of China (No 81370452).

\section{Disclosure}

The authors report no conflicts of interest in this work.

\section{References}

1. Eknoyan G, Lameire N, Eckardt K, Kasiske B. Kidney disease: improving global outcomes (KDIGO) acute kidney injury work group. KDIGO clinical practice guideline for acute kidney injury. Kidney Int Suppl. 2012;2:1-138.

2. Chao C-T, Tsai H-B, Lin Y-F, Ko W-J. Acute kidney injury in the elderly: only the tip of the iceberg. J Clin Gerontol Geriatr. 2014;5(1): $7-12$.

3. Chronopoulos A, Cruz DN, Ronco C. Hospital-acquired acute kidney injury in the elderly. Nat Rev Nephrol. 2010;6(3):141-149.

4. Anderson S, Eldadah B, Halter JB, et al. Acute kidney injury in older adults. J Am Soc Nephrol. 2011;22(1):28-38.

5. Chao CT, Tsai HB, Wu CY, et al. The severity of initial acute kidney injury at admission of geriatric patients significantly correlates with subsequent in-hospital complications. Sci Rep. 2015;5: 13925.

6. Chao CT, Lin YF, Tsai HB, Wu VC, Ko WJ. Acute kidney injury network staging in geriatric postoperative acute kidney injury patients: shortcomings and improvements. J Am Coll Surg. 2013;217(2):240-250.

7. Ali T, Khan I, Simpson W, et al. Incidence and outcomes in acute kidney injury: a comprehensive population-based study. J Am Soc Nephrol. 2007; 18(4):1292-1298. 
8. Akposso K, Hertig A, Couprie R, et al. Acute renal failure in patients over 80 years old: 25-years' experience. Intensive Care Med. 2000;26(4): 400-406.

9. Bouchard J, Soroko SB, Chertow GM, et al. Fluid accumulation, survival and recovery of kidney function in critically ill patients with acute kidney injury. Kidney Int. 2009;76(4):422-427.

10. Siew ED, Ware LB, Ikizler TA. Biological markers of acute kidney injury. J Am Soc Nephrol. 2011;22(5):810-820.

11. Waikar SS, Bonventre JV. Creatinine kinetics and the definition of acute kidney injury. J Am Soc Nephrol. 2009;20(3):672-679.

12. Mehta RL, McDonald B, Gabbai F, et al. Nephrology consultation in acute renal failure: does timing matter? Am J Med. 2002;113(6):456-461.

13. Ponce D, Zorzenon Cde P, dos Santos NY, Balbi AL. Early nephrology consultation can have an impact on outcome of acute kidney injury patients. Nephrol Dial Transplant. 2011;26(10):3202-3206.

14. Perez-Valdivieso JR, Bes-Rastrollo M, Monedero P, de Irala J, Lavilla FJ. Prognosis and serum creatinine levels in acute renal failure at the time of nephrology consultation: an observational cohort study. BMC Nephrol. 2007;8:14.
15. Meier P, Bonfils RM, Vogt B, Burnand B, Burnier M. Referral patterns and outcomes in noncritically ill patients with hospital-acquired acute kidney injury. Clin J Am Soc Nephrol. 2011;6(9):2215-2225.

16. Balasubramanian G, Al-Aly Z, Moiz A, et al. Early nephrologist involvement in hospital-acquired acute kidney injury: a pilot study. Am J Kidney Dis. 2011;57(2):228-234.

17. Levey AS, Stevens LA, Schmid CH, et al. A new equation to estimate glomerular filtration rate. Ann Intern Med. 2009;150(9):604-612.

18. Levin A, Stevens PE. Summary of KDIGO 2012 CKD guideline: behind the scenes, need for guidance, and a framework for moving forward. Kidney Int. 2014;85(1):49-61.

19. Costa e Silva VT, Liano F, Muriel A, Diez R, de Castro I, Yu L. Nephrology referral and outcomes in critically ill acute kidney injury patients. PLoS One. 2013;8(8):e70482.
Clinical Interventions in Aging

\section{Publish your work in this journal}

Clinical Interventions in Aging is an international, peer-reviewed journal focusing on evidence-based reports on the value or lack thereof of treatments intended to prevent or delay the onset of maladaptive correlates of aging in human beings. This journal is indexed on PubMed Central, MedLine,

\section{Dovepress}

CAS, Scopus and the Elsevier Bibliographic databases. The manuscript management system is completely online and includes a very quick and fair peer-review system, which is all easy to use. Visit http://www.dovepress. com/testimonials.php to read real quotes from published authors. 